\title{
Nuclear Quantum Effects in Water at the Triple Point: Using Theory as a
} Link Between Experiments Supporting Information

\author{
Bingqing Cheng, ${ }^{\dagger}$ Jörg Behler, ${ }^{\ddagger}$ and Michele Ceriotti ${ }^{*} \dagger$ \\ Laboratory of Computational Science and Modelling, Institute of Materials, Ecole \\ Polytechnique Fédérale de Lausanne, Lausanne, Switzerland, and Lehrstuhl für \\ Theoretische Chemie, Ruhr-Universität Bochum, Bochum, Germany \\ E-mail: michele.ceriotti@epfl.ch
}

\section{Construction of the neural network potential}

The high-dimensional neural network potential for water, which is flexible and fully dissociable, has been constructed using the method proposed by Behler and Parrinello. ${ }^{1}$ In this approach the total energy of the system is determined as a sum of environment-dependent atomic energies. For each atom in the system, the positions of all neighboring atoms inside a cutoff radius, which has to be converged, are described by a set of atom-centered many-body symmetry functions. ${ }^{2}$ These sets of function values are then used as input vectors for the atomic neural networks yielding the atomic energy contributions. Consequently, the analytic

\footnotetext{
*To whom correspondence should be addressed

${ }^{\dagger}$ Laboratory of Computational Science and Modelling, Institute of Materials, Ecole Polytechnique Fédérale de Lausanne, Lausanne, Switzerland

${ }^{\ddagger}$ Lehrstuhl für Theoretische Chemie, Ruhr-Universität Bochum, Bochum, Germany
} 
total energy expression is a sum over the outputs of all individual atomic neural networks, and analytic gradients for the calculation of the forces are readily available. Further details on the method can be found in two recent reviews. ${ }^{3,4}$ The NN potential has been constructed using the program RuNNer, ${ }^{5}$ while the simulations employing this potential have been carried out using a modified version ${ }^{6}$ of the LAMMPS code. ${ }^{7}$

For describing the energetically relevant atomic environments, we have used the symmetry function sets for hydrogen (27 functions) and oxygen (30 functions) atoms employing a cutoff radius of 12.0 a.u., which have been determined by Morawietz et al. ${ }^{8}$ in previous work on neural network potentials for water employing GGA-DFT reference data. Here, we use energies and forces of the liquid and ice phases of water computed at the hybrid density functional theory level (B3LYP functional ${ }^{9}$ with D3 dispersion corrections ${ }^{10}$ ) using the CP2K code. ${ }^{11}$ Computational details of the reference B3LYP calculations were the same as those used in Ref. ${ }^{12}$ and. ${ }^{13}$

In total, we have calculated 25,690 reference bulk structures including between 64 and 96 water molecules. This set has been split into a training set of 20,650 structures to determine the NN parameters, and an independent test set of 5,040 structures to estimate the quality of the NN potential for atomic configurations not included in the training set. In the optimization of the 676 and 721 parameters of the hydrogen and oxygen atomic NNs, respectively, which consist of two hidden layers with 15 nodes each, we have used the total energies and all force components present in the training set resulting in more than 12 million pieces of information. The resulting root mean squared errors (RMSE) of the energies in the training and the test set are 0.46 and $0.47 \mathrm{meV} /$ atom, respectively, while the RMSE values of the forces in both sets are $0.054 \mathrm{eV} /$ bohr. 


\section{A calibration curve for $\left\langle E_{k}\right\rangle$ in liquid water from the triple point to the critical point}

In the main text of the present study we argued that the isotope fractionation ratios between different phases of water are closely related to the mean nuclear kinetic energies $\left\langle E_{k}\right\rangle$. In particular, within a quasi-harmonic description of the vibrational modes of vapor and liquid water, the mean kinetic energies of atoms in the liquid phase can be estimated from knowledge of the gas-phase value and the fractionation ratio. Using H/D fractionation,

$$
\left\langle E_{k}\right\rangle_{\mathrm{L}}(\mathrm{H}) \approx\left\langle E_{k}\right\rangle_{\mathrm{V}}(\mathrm{H})+\frac{k_{B} T \ln \alpha_{\mathrm{L}-\mathrm{V}}}{2\left(1-\sqrt{m_{\mathrm{H}} / m_{\mathrm{D}}}\right)} .
$$

Furthermore, MB-pol in the gas phase uses the most accurate force field available for a single water molecule, ${ }^{15}$ that is based on the highest levels of theory. It is reasonable to state that $\left\langle E_{k}\right\rangle$ of water vapor computed from converged PIMD simulations based on such potential is essentially exact. Based on these assumptions, one can estimate the kinetic energies of $\mathrm{O}$ and $\mathrm{H}$ in liquid water from $\left\langle E_{k}\right\rangle_{\mathrm{V}}$ and experimental fractionation data. To do that, we performed PIMD simulation using 64 beads for gas phase water at different temperatures and densities along the coexistence line in the water phase diagram, with other simulation details as specified in the main text. At $T \geq 500 \mathrm{~K}$, the simulation cell has 9 water molecules at the experimental vapor density at coexistence pressure, otherwise the system comprises an individual molecule in vacuum. The $\left\langle E_{k}\right\rangle_{\mathrm{V}}$ computed from PIMD simulation using MB-pol, together with $\left\langle E_{k}\right\rangle_{\mathrm{L}}$ estimated using experimental fractionation values, ${ }^{14}$ are reported in Figure 1. For ease of reference, individual values with their error bars are reported in Table 1. 


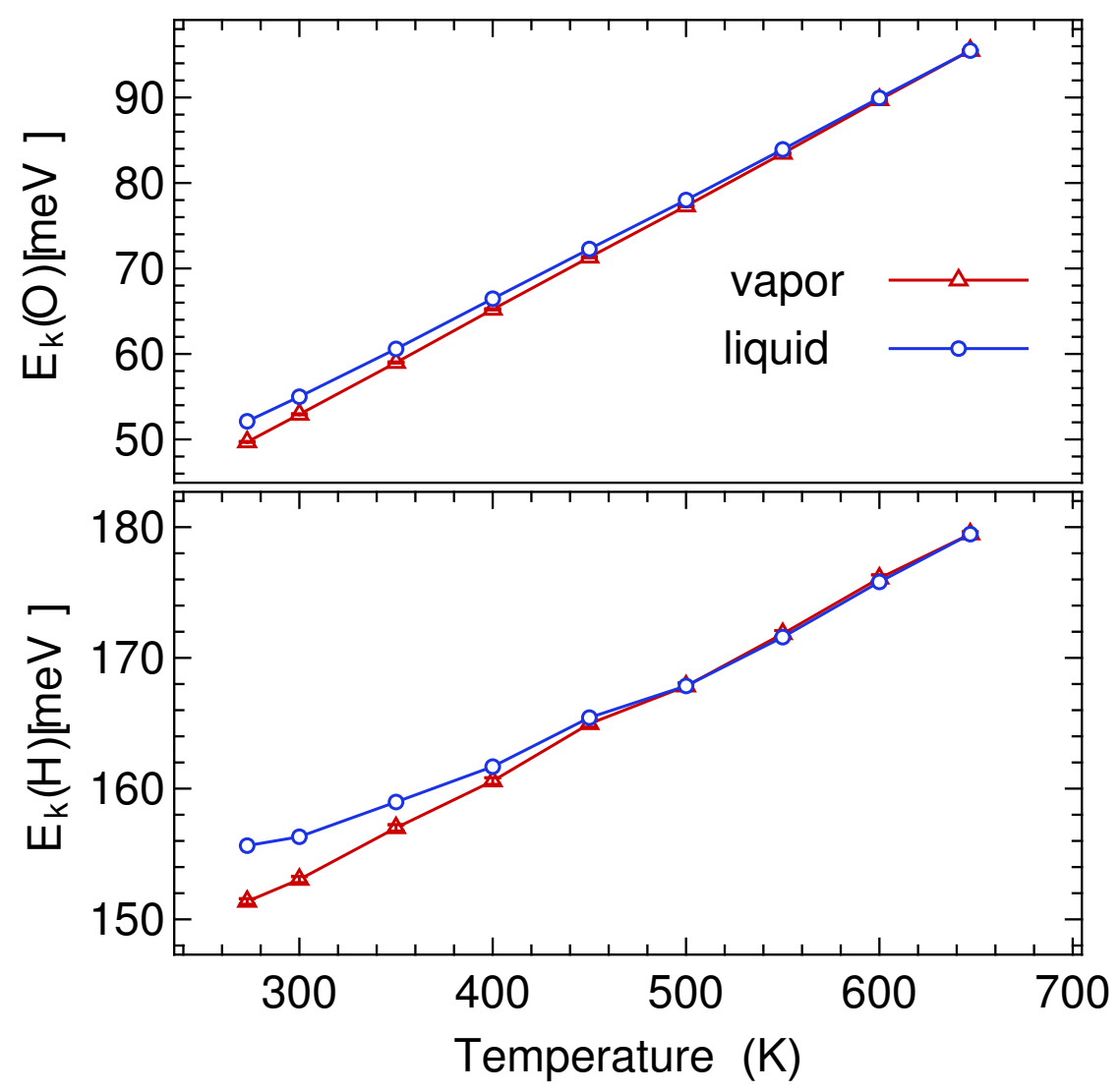

Figure 1: The mean kinetic energies of $\mathrm{O}$ and $\mathrm{H}$ in vapor and liquid phases of water from the triple point to the critical point along coexistence line. The statistical uncertainties for the kinetic energies of vapor computed from PIMD simulations are smaller than the symbols. The kinetic energies of liquid water were obtained based on the isotope fractionation data in Ref. ${ }^{14}$ and Eq. 1

Table 1: The mean kinetic energies of $\mathrm{O}$ and $\mathrm{H}$ in vapor and liquid phases of water, and experimental isotope fractionation data from Ref. ${ }^{14}$

\begin{tabular}{l|lll|lll}
\hline \hline $\begin{array}{l}\text { Temperature } \\
{[\mathrm{K}]}\end{array}$ & $\left\langle E_{k}\right\rangle_{\mathrm{V}}(H)$ & $\begin{array}{l}\left\langle E_{k}\right\rangle_{\mathrm{L}}(H) \\
{[\mathrm{EST}}\end{array}$ & $\begin{array}{l}1000 \ln \left(\alpha_{\mathrm{L}-\mathrm{V}}\right) \\
\mathrm{HIMD}[\mathrm{meV}]\end{array}$ & $\begin{array}{l}\left\langle E_{k}\right\rangle_{\mathrm{V}}(O) \\
\mathrm{PIMD}[\mathrm{meV}]\end{array}$ & $\begin{array}{l}\left\langle E_{k}\right\rangle_{\mathrm{L}}(O) \\
{[\mathrm{meV}]}\end{array}$ & $\begin{array}{c}\mathrm{EST} \\
1000 \ln \left(\alpha_{\mathrm{L}-\mathrm{V})}\right) \\
16\end{array}$ \\
\hline 273 & $151.4 \pm 0.2$ & 155.6 & 106.2 & $49.70 \pm 0.05$ & 52.12 & 11.77 \\
300 & $153.1 \pm 0.2$ & 156.3 & 74.0 & $52.93 \pm 0.06$ & 55.00 & 9.15 \\
350 & $157.0 \pm 0.2$ & 159.0 & 38.3 & $58.99 \pm 0.07$ & 60.59 & 6.06 \\
400 & $160.6 \pm 0.2$ & 161.7 & 18.7 & $65.21 \pm 0.07$ & 66.46 & 4.16 \\
450 & $165.0 \pm 0.2$ & 165.4 & 7.07 & $71.31 \pm 0.07$ & 72.28 & 2.85 \\
500 & $167.8 \pm 0.2$ & 167.9 & 0.20 & $77.30 \pm 0.07$ & 78.01 & 1.88 \\
550 & $172.2 \pm 0.2$ & 171.6 & -3.14 & $83.46 \pm 0.07$ & 83.92 & 1.12 \\
600 & $176.1 \pm 0.3$ & 175.8 & -3.19 & $89.73 \pm 0.07$ & 89.96 & 0.50 \\
647 & $179.5 \pm 0.2$ & 179.5 & 0.00 & $95.49 \pm 0.05$ & 95.49 & 0.00 \\
\hline \hline
\end{tabular}




\section{A comparison between simulation and experimental iso- tope fractionation data}

In the Figure 1 of the main text of the present study, we illustrated the isotope fractionation ratio of $\mathrm{H} / \mathrm{D}$ and ${ }^{16} \mathrm{O} /{ }^{18} \mathrm{O}$ between liquid and vapor phases, and between ice and liquid phases of water. For ease of reference, in Table 2 and 3 we quote the individual values with error bars.

Table 2: The isotope fractionation ratio of $\mathrm{H} / \mathrm{D}$ between liquid and vapor phases (L-V), between solid and liquid phases (S-L), and between solid and gas phases (S-G) of water. The experimental (EXP) values are taken from a number of studies. ${ }^{14,16-19}$

\begin{tabular}{|c|c|c|c|c|c|}
\hline system & $273 \mathrm{~K}$ L-G & $273 \mathrm{~S}-\mathrm{L}$ & $273 \mathrm{~S}-\mathrm{G}$ & $300 \mathrm{~K} \mathrm{~L}-\mathrm{G}$ & $580 \mathrm{~K} \mathrm{~L}-\mathrm{G}$ \\
\hline q-TIP4p(F) & $145 \pm 2$ & $23 \pm 3$ & $168 \pm 2$ & $111 \pm 3$ & $-3 \pm 1$ \\
\hline MB-Pol & $144 \pm 7$ & $9 \pm 8$ & $153 \pm 5$ & $92 \pm 7$ & $5 \pm 3$ \\
\hline NN & $110 \pm 5$ & $21 \pm 5$ & $131 \pm 3$ & $82 \pm 5$ & - \\
\hline \multirow[t]{4}{*}{ Experiment } & 106.43 (Majoube) & $18.53 \quad$ (Ellehoi) & 124.96 (Ellehoi) & 73.97 (Horita) & -3.57 (Horita) \\
\hline & 106.19 (Horita) & 20.59 (Arnason) & & & \\
\hline & & $19.31 \quad$ (ONeil) & & & \\
\hline & & 23.23 (Merlivat) & & & \\
\hline
\end{tabular}

Table 3: The isotope fractionation ratio of ${ }^{16} \mathrm{O} /{ }^{18} \mathrm{O}$ between liquid and vapor phases (L-V), between solid and liquid phases (S-L), and between solid and gas phases (S-G) of water. The experimental (EXP) values are taken from a number of studies. ${ }^{14,16-19}$

\begin{tabular}{l|lll|ll}
\hline \hline system & $273 \mathrm{~K} \mathrm{L-G}$ & $273 \mathrm{~S}-\mathrm{L}$ & $273 \mathrm{~S}-\mathrm{G}$ & $300 \mathrm{~K}$ L-G & $580 \mathrm{~K}$ L-G \\
\hline q-TIP4p(F) & $16.79 \pm 0.04$ & $2.39 \pm 0.01$ & $19.17 \pm 0.03$ & $13.29 \pm 0.02$ & $1.87 \pm 0.01$ \\
MB-Pol & $11.88 \pm 0.18$ & $2.89 \pm 0.07$ & $14.77 \pm 0.15$ & $8.61 \pm 0.08$ & $0.95 \pm 0.01$ \\
NN & $15.28 \pm 0.06$ & $0.53 \pm 0.15$ & $15.81 \pm 0.17$ & $12.67 \pm 0.09$ & - \\
Experiment & 11.63 (Majoube) & $2.77 \quad$ (Ellehoi) & 14.40 (Ellehoi) & 9.15 (Horita) & 0.73 (Horita) \\
& 11.77 (Horita) & 3.00 (ONeil) & & & \\
& & 3.40 (Majoube) & & \\
\hline \hline
\end{tabular}

\section{References}

(1) Behler, J.; Parrinello, M. Phys. Rev. Lett. 2007, 98, 146401.

(2) Behler, J. J. Chem. Phys. 2011, 134, 074106.

(3) Behler, J. J. Phys.: Condens. Matter 2014, 26, 183001. 
(4) Behler, J. Int. J. Quantum Chem. 2015, 115, 1032.

(5) Behler, J. RuNNer - A neural network program for constructing high-dimensional neural network potentials; Ruhr-Universität Bochum, 2007-2016.

(6) Singraber, A.; Morawietz, T.; Behler, J.; Dellago, C. to be published

(7) Plimpton, S. J. Comp. Phys. 1995, 117, 1.

(8) Morawietz, T.; Singraber, A.; Dellago, C.; Behler, J. submitted 2016,

(9) Becke, A. D. J. Chem. Phys. 1993, 98, 5648.

(10) Grimme, S.; Antony, J.; Ehrlich, S.; Krieg, H. The Journal of Chemical Physics 2010, 132, 154104 .

(11) VandeVondele, J.; Krack, M.; Mohamed, F.; Parrinello, M.; Chassaing, T.; Hutter, J. Comp. Phys. Comm. 2005, 167, 103-128.

(12) Wang, L.; Ceriotti, M.; Markland, T. E. J. Chem. Phys. 2014, 141, 104502.

(13) Gasparotto, P.; Hassanali, A. A.; Ceriotti, M. J. Chem. Theory Comput. 2016, 12, $1953-1964$.

(14) Horita, J.; Wesolowski, D. J. Geochimica et Cosmochimica Acta 1994, 58, 3425-3437.

(15) Partridge, H.; Schwenke, D. W. J. Chem. Phys. 1997, 106, 4618.

(16) Merlivat, L.; Nief, G. Tellus A 1967, 19.

(17) Majoube, M. Nature 1970, 226, 1242-1242.

(18) O'Neil, J. R. The Journal of Physical Chemistry 1968, 72, 3683-3684.

(19) Ellehoj, M.; Steen-Larsen, H. C.; Johnsen, S. J.; Madsen, M. B. Rapid Communications in Mass Spectrometry 2013, 27, 2149-2158. 